\title{
A Simple Proof of Damien's Theorem and Duality in Theory of the Zero-Distance Phase Front
}

\author{
Andrey Valerievich Gitin \\ Institut für Optik und Atomare Physik, Technische Universität Berlin, Sekretariat ER 1-1, Strasse des 17. Juni 135, 10623 Berlin, Germany \\ Correspondence should be addressed to Andrey Valerievich Gitin; andrey.gitin@gmx.de \\ Received 9 April 2015; Accepted 31 May 2015 \\ Academic Editor: Giuseppe Castaldi \\ Copyright ( 2015 Andrey Valerievich Gitin. This is an open access article distributed under the Creative Commons Attribution \\ License, which permits unrestricted use, distribution, and reproduction in any medium, provided the original work is properly \\ cited. \\ Duality plays the main role in all mathematical theories. In wave optics based on the concept of "the zero-distance phase front," \\ duality takes the form of Damien's theorem and the mirror symmetry between the conic refracting surfaces with the plane zero- \\ distance phase front and the plane refracting surfaces with the conic zero-distance phase front. A systematic study of these dualities \\ has been performed.
}

\section{Introduction}

In 1825 Joseph Gergonne [1] noticed the "symmetry" of roles played by points and lines in definitions and theorems of geometry: if we assume that all parallel lines intersect at infinity (the axiom of projective geometry), any theorem or definition one-to-one corresponds to another theorem or definition (the so-called dual statement), when we substitute "point" for "line," "lie on" for "pass through," "collinear" for "concurrent," "intersection" for "join," or vice versa. At present, various types of duality have been discovered in various branches of mathematics and theoretical physics (including optics $[2,3]$ ).

It is known that the application of the law of refraction together with the axioms of geometry gives rise to "optical geometry," implemented in design of aspheric lenses [4]. In optical geometry based on the concept of "the zero-distance phase front" [5] duality takes the form of Damien's theorem and of the mirror symmetry between the conic refracting surfaces with the plane zero-distance phase front and the plane refracting surfaces with the conic zero-distance phase front [4, 6-11].

We propose a simple proof of Damien's theorem and describe dual pairs: "the refracting (reflecting) surface-the zero-distance phase front."

\section{Refraction and Reflection}

A monochromatic wave can be described by a locus of points of the wave with the same phase of oscillation, the so-called phase front [12]. The wave propagation can be described by a family of the phase fronts. An alternative way of describing of wave propagation is given by orthogonal trajectories to the phase fronts, the so-called light rays. The shape of the phase fronts (and light rays) is usually determined by the geometry of the source and the properties of the medium. In a homogeneous medium a point source $S$ generates a family of concentric spherical (circular) phase fronts with a common center at $S$ (or a homocentric beam of light rays with a vertex at $S$ ) (Figure 1(a)), and a plane source generates plane phase fronts (or parallel rays) (Figure 1(b)). In an inhomogeneous medium phase fronts can be very complicated, however the principle of equal optical paths [12] states that, in an optical system consisting of refracting and reflecting elements, the optical path length (OPL) between any two phase fronts is identically the same for all rays (Figure 2). The OPL is defined by the line integral along any ray path $\gamma$ :

$$
\operatorname{OPL}[\gamma(\omega)]=\int_{\gamma} n(x, y, z ; \omega) d s,
$$




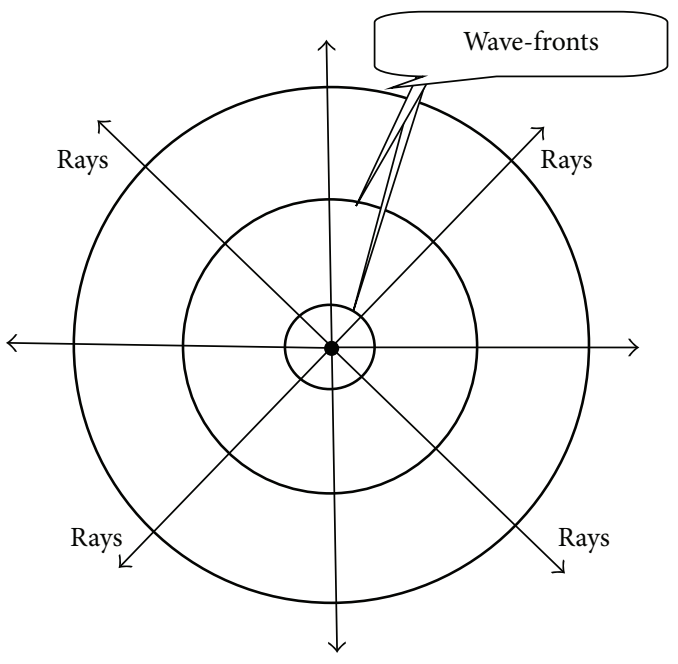

(a)

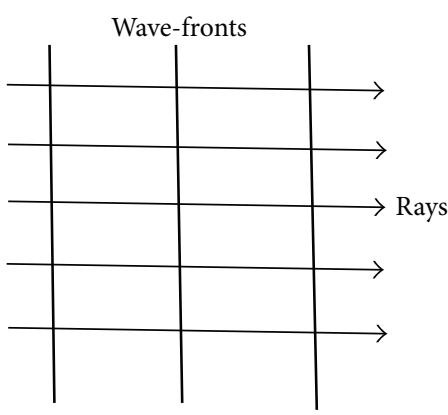

(b)

Figure 1: Spherical (a) and plane (b) phase fronts in a homogeneous and isotropic medium.

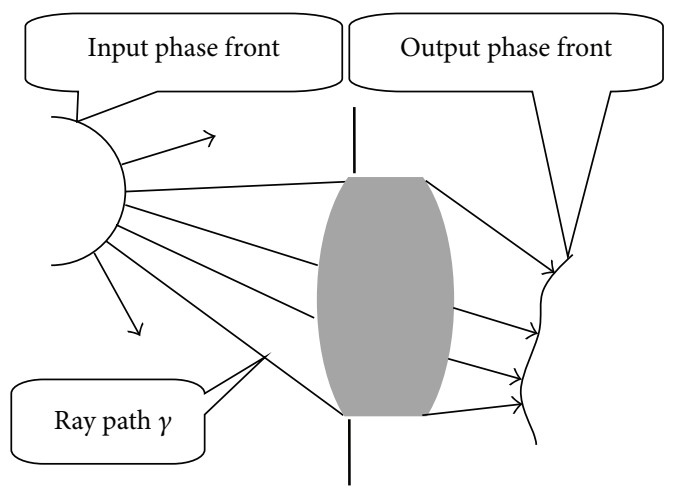

FIgURE 2: The principle of equal optical paths.

where $n(x, y, z)$ is the refraction index at each point along the ray path $\gamma$ and $d s^{2}=d x^{2}+d y^{2}+d z^{2}$.

Let us consider the interaction of a spherical wave from a point source $S$ with a single refracting (or reflecting) surface $g$ separating two media with refraction indexes $n_{1}$ and $n_{2}$ at a distance $d=0 S$ to the right of it (Figure 3(a)). For simplicity, but with no loss of generality, let us assume that two closely adjacent rays from a source $S$ are focused by the refracting surface at a point $F$, where the refracting surface is an axially

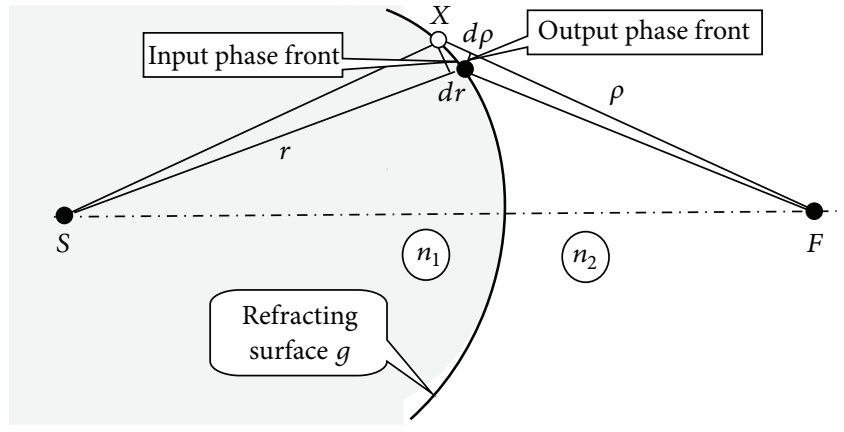

(a)

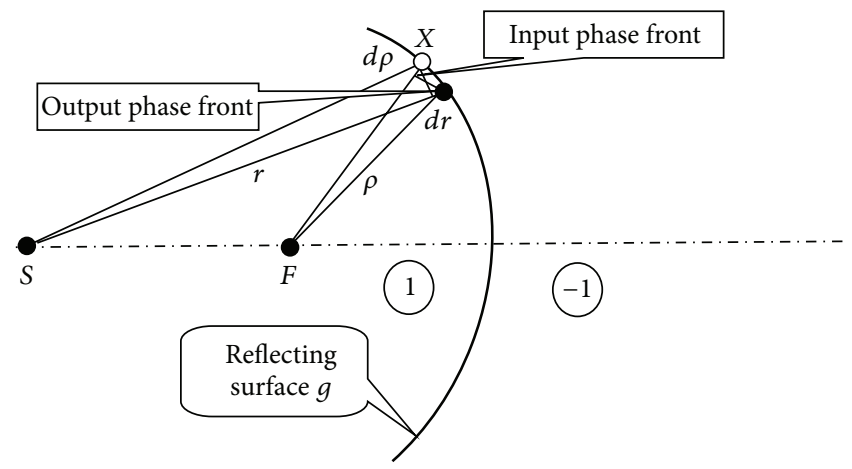

(b)

FIgURE 3: (a) The law of refraction and (b) the law of reflection from the point of view of the principle of equal optical paths.

symmetric surface, and that the axis of symmetry $0 z$ passes through points $S$ and $F$ [6-9]. A plane containing the axis $0 z$ is a meridional plane. In this plane the curve $g$ represents a refracting surface, closely adjacent rays are incident upon the plane $g$ in a neighborhood of the point $X$, and $r$ and $\rho$ are bipolar coordinates of $X$ with respect to $S$ and $F$.

In case of the refracting surface $g$ (Figure 3(a)) the principle of equal optical paths takes the form [6-9]

$$
n_{1} \cdot d r=n_{2} \cdot d \rho
$$

According to the theorem of Malus and Dupin $[6-9,12]$ the normal congruence between light rays and phase fronts will remain the normal congruence after refraction on $g$. Thus, $d r$ and $d \rho$ are the opposites of the two right triangles with the common hypotenuse, so that the angles $\alpha$ and $\beta$ are related by the equation

$$
n_{2} \sin \beta=n_{1} \sin \alpha,
$$

where $\alpha$ and $\beta$ are the angles between the incident and the refracted phase fronts with the surface $g$ at $X$. As rays are the normals to the phase fronts, the angles $\alpha$ and $\beta$ can be interpreted as the angles between the incident and the refracted rays with the normal to the surface $g$ at $X$ (Figure 3(a)). Equation (3) describes Snell's law of refraction.

Lemma 1. The incident angle $\alpha$ (and therefore the refracted angle $\beta$ ) is uniquely determined by the difference between the incident and the refracted angles: $\Delta \equiv \alpha-\beta$ (Figure 4(a)). 


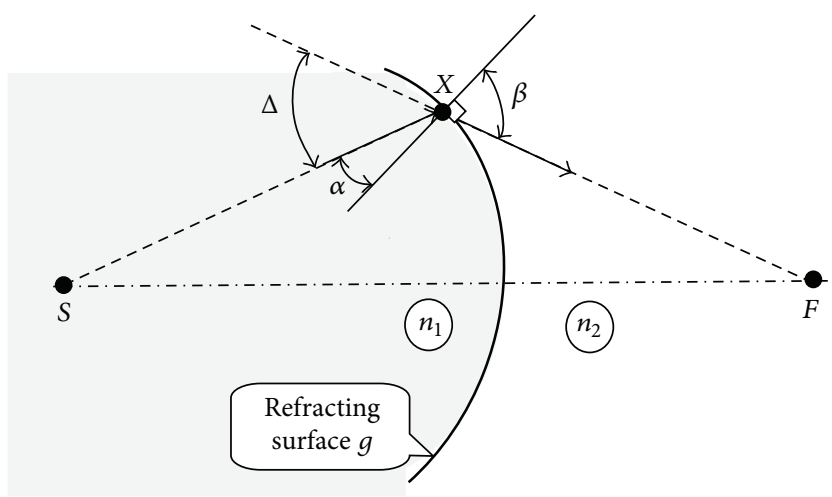

(a)

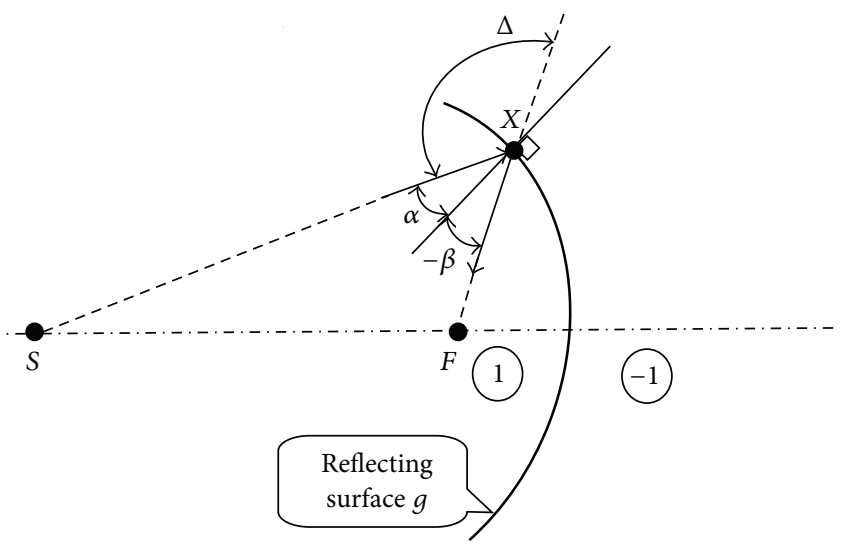

(b)

Figure 4: (a) The law of refraction and (b) the law of reflection from the point of view of ray optics.

Indeed, from Snell's law (3), $n_{2} \sin (\alpha+\Delta)=n_{1} \sin \alpha$, and the identity, $\sin (\alpha+\Delta)=\sin \alpha \cdot \cos \Delta+\cos \alpha \cdot \sin \Delta$, we easily obtain the incident angle $\alpha=\operatorname{arctg} g\left(\sin \Delta /\left(n_{1} / n_{2}-\cos \Delta\right)\right)$ and the angle of reflection $\beta=\alpha+\Delta$.

Let us design a refracting surface $g$ which transforms a divergent beam of rays (spherical wave) radiated from the on-axis source $S$ into a plane-parallel beam of rays (a plane wave). Let the axis $x$ be perpendicular to the axis of symmetry (Figures 5(a) and 5(b)). From (2) we have

$$
n_{1} \cdot d r=n_{2} \cdot d x
$$

where $x=r \cdot \cos \theta$. Direct integration of (4) leads to

$$
r=\frac{n_{2}}{n_{1}} r \cdot \cos \theta+\text { constant }
$$

The constant is being evaluated by the condition $r=q$ when $\theta=0$. So (5) gives the conic section:

$$
r=\frac{l}{1-\varepsilon \cdot \cos \theta}=\frac{q\left(1-n_{2} / n_{1}\right)}{1-\left(n_{2} / n_{1}\right) \cdot \cos \theta},
$$

where $\varepsilon \equiv n_{2} / n_{1}$ and $l \equiv q(1-\varepsilon)$. The source is located at the focus farthest from the conic surface.
In case of the reflecting surface $g$ (Figure 3(b)), the principle of equal optical paths takes the form

$$
d r=-d \rho
$$

or

$$
\sin \beta=-\sin \alpha,
$$

where $\alpha$ and $\beta$ are the angles between the incident and the reflected phase fronts with the surface $g$ at $X$. As rays are the normals to the phase fronts, the angles $\alpha$ and $\beta$ can be interpreted as the angles between the incident and the reflected rays with the normal to the surface $g$ at $X$ (Figure 4(b)). Equation (8) describes the law of reflection; $\beta=-\alpha$. Note, that (8) is (3) if we put $n_{1}=1$ and $n_{2}=-1$.

Lemma 2. The incident angle $\alpha$ (and therefore the reflected angle $\beta$ ) is uniquely determined by the difference between the incident and the reflected angles: $\Delta \equiv \pi-(\alpha-\beta)$ (Figure $4(b)$ ). Indeed, from the law of reflection (2) we easily obtain the incident angle $\alpha=(\pi-\Delta) / 2$ and the angle of reflection $\beta=-\alpha$.

Let us design a reflecting surface $g$ which transforms a divergent beam of rays (a spherical wave) radiated from the source $S$ into a plane-parallel beam of rays (a plane wave) (Figure 5(c)). In this case from (7) we have

$$
d r=-d x,
$$

where $x=r \cdot \cos \theta$. Direct integration of (9) leads to

$$
r=-r \cdot \cos \theta+\text { constant }
$$

The constant is being evaluated by the condition $r=f$ when $\theta=0$. So (10) gives a parabola with a focal length $d$ :

$$
r=\frac{2 f}{1+\cos \theta} .
$$

\section{Zero-Distance Phase Front}

Phase fronts will be some changing surfaces: at one time they can be completely smooth but at the next moment they have cusps or other singular points [11]. It is known that from a family of phase fronts can be extracted one in the simplest form which is a representative of the entire family nevertheless [11]. This phase front is the so-called zero-distance phase front [4-9].

Let us continue the refracted ray in its reverse direction to the point $P$, where

$$
n_{2}|X P|=-n_{1}|S X|,
$$

the negative sign indicating the reverse direction. As point $X$ moves over the surface $g$, point $P$ moves over a surface $h$. This surface $h$ is the locus of points $\{P\}$, which is the desired zerodistance phase front (Figure 6(a)). The zero-distance phase front replaces the point source $S$ and the refracting surface $g$, so that this replacement does not affect the family of wavefronts in the second medium. 


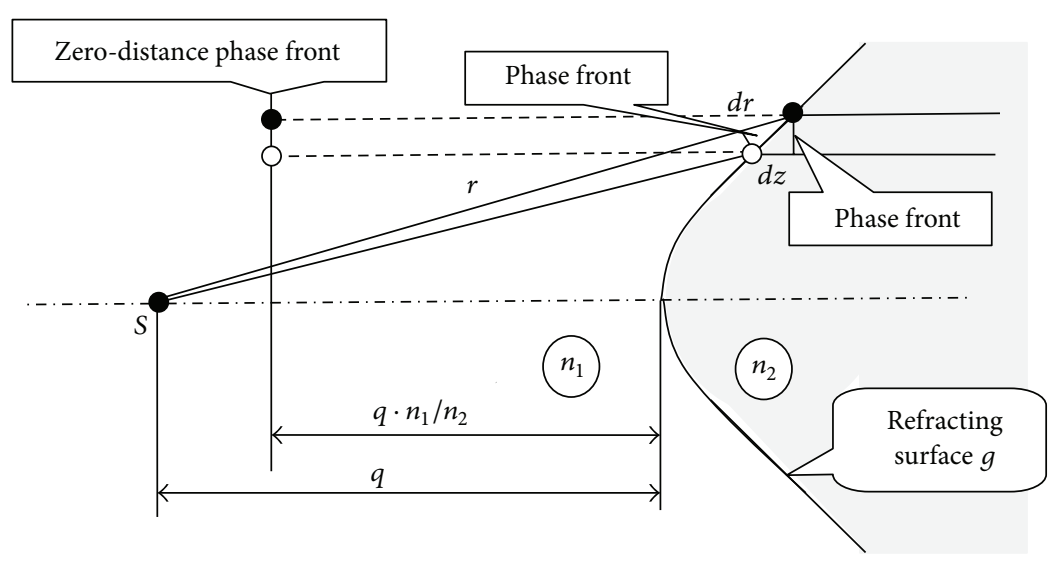

(a)

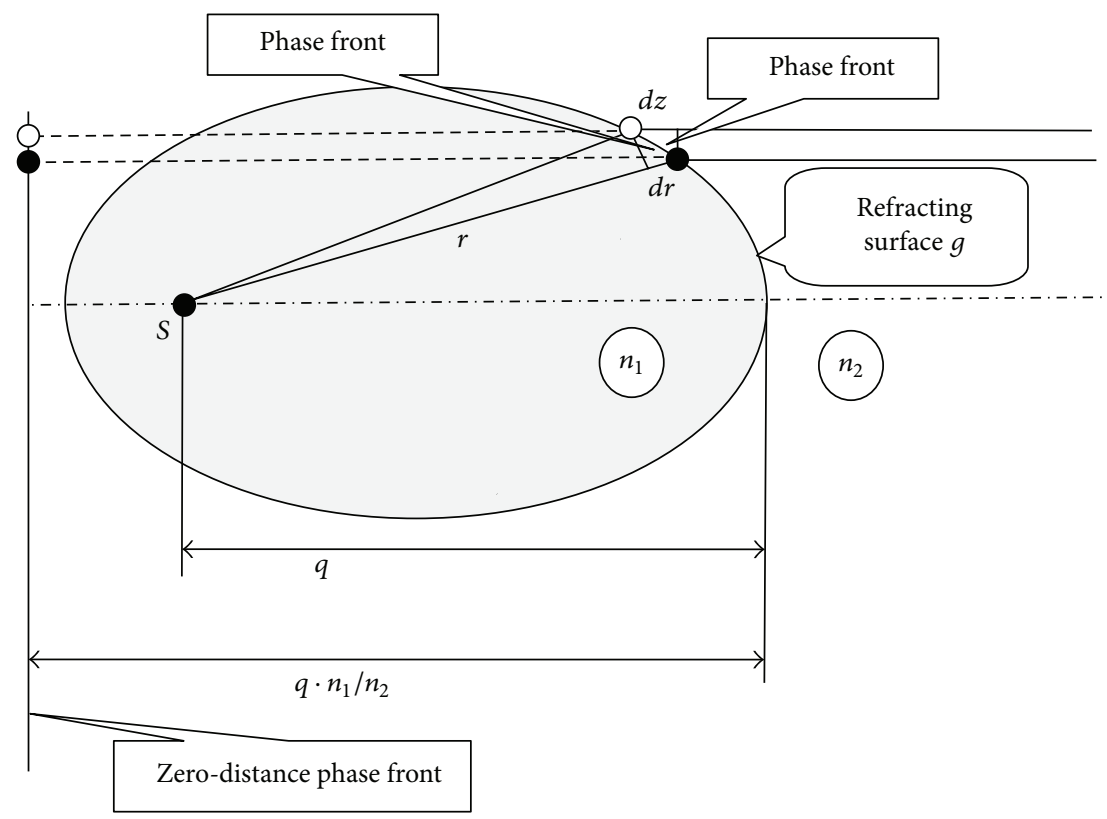

(b)

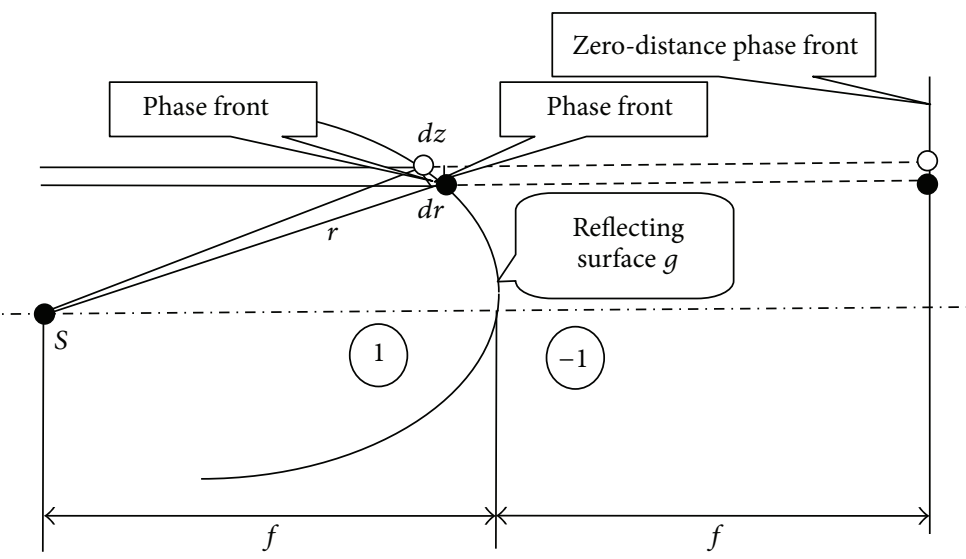

(c)

Figure 5: "Designing" the conic optical surfaces. (a) Hyperbolic refracting surface $\left(n_{1}<n_{2}\right)$. (b) Elliptical refracting surface $\left(n_{1}>n_{2}\right)$. (c) Parabolic reflecting surface $\left(n_{1}=1, n_{2}=-1\right)$. 


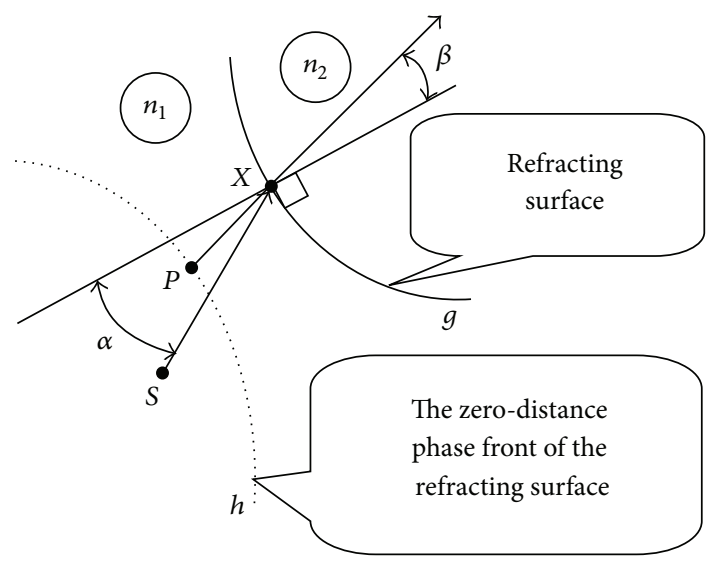

(a)

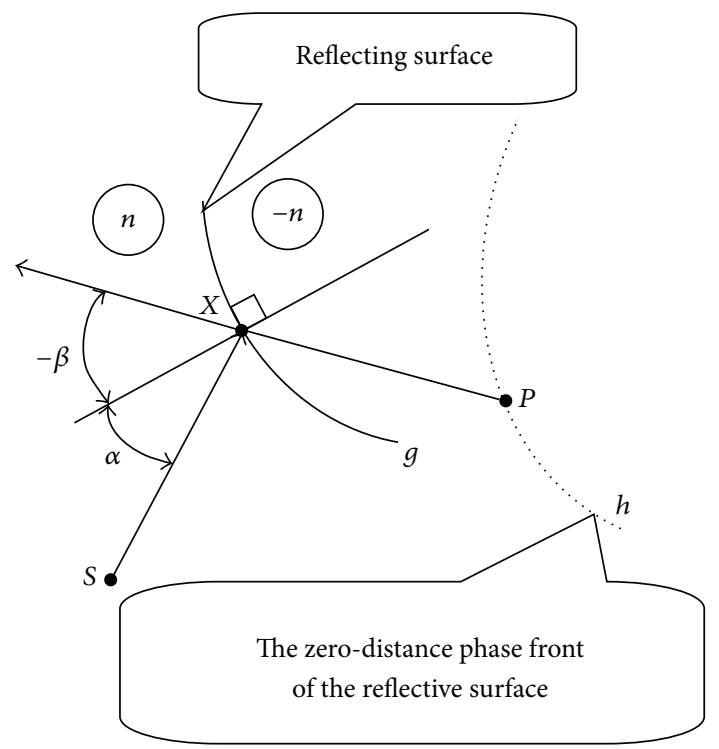

(b)

Figure 6: The zero-distance phase front $h=\{P\}$ with respect to vertex $S$ of the homocentric beam of light rays. (a) The ray refracted at point $X$ of the surface $g$ goes in the opposite direction for the distance $\left(n_{2} / n_{1}\right)|S X|$ to the point $P$. (b) The ray reflected at point $X$ of the surface $g$ goes in the opposite direction for the distance $|S X|$ to the point $P$.

For the case of a reflecting surface $g$ we put $n_{1}=n$ and $n_{2}=-n$ in (12) (Figure 6(b)). Therefore $X P$ is continued in the forward direction that is on the side of the reflector opposite to the source $S$, and

$$
|X P|=|S X| \text {. }
$$

Note that the zero-distance phase front of a parallel beam of rays is a plane (Figures 5(a), 5(b), and 5(c)). Thus, we have the following.

If $n_{2}>n_{1}$ (the refraction occurs from a less dense medium to a more dense medium), the single refracting surface giving the plane zero-distance phase front is a hyperbolic surface (Figure 5(a)).

If $n_{2}<n_{1}$ (the refraction occurs from a more dense medium to a less dense medium), the single refracting surface giving the plane zero-distance phase front is an elliptical surface (Figure 5(b)).

If $n_{1}=1$ and $n_{2}=-1$, the single reflecting surface giving the plane zero-distance phase front is a parabolic surface (Figure 5(c)).

Let us consider the case in which the refracting surface $g$ is a plane surface [11]. If $l=|S X|$ is the length of this ray from $S(-q, 0)$ to $X(0, y)$ and $\beta$ is the refracted angle, the point $P$ of the zero-distance phase front (12) has coordinates

$$
\begin{aligned}
& \bar{y}=y-\frac{n_{1}}{n_{2}} l \cdot \sin \beta, \\
& \bar{x}=-\frac{n_{1}}{n_{2}} l \cdot \cos \beta .
\end{aligned}
$$

The relationship between the angle of incidence $\alpha$ and the angle of refraction $\beta$ is given by Snell's law (3). The height of $X$ with respect to 0 and the length of the ray from $S$ to $X$ are given by

$$
\begin{aligned}
& y=q \cdot \tan \alpha, \\
& l=\frac{q}{\cos \alpha} .
\end{aligned}
$$

Substituting the previous relations, we obtain

$$
\begin{aligned}
& \bar{y}=\left[1-\left(\frac{n_{1}}{n_{2}}\right)^{2}\right] q \cdot \tan \alpha, \\
& \bar{x}=-\frac{q}{\cos \alpha} \cdot \frac{n_{1}}{n_{2}} \sqrt{1-\left(\frac{n_{1}}{n_{2}}\right)^{2} \sin ^{2} \alpha .}
\end{aligned}
$$

Eliminating $\alpha$ between these two expressions and using the identity $1+\tan ^{2} \alpha=1 / \cos ^{2} \alpha$, we obtain

$$
\left(\frac{\bar{x}}{q \cdot\left(n_{1} / n_{2}\right)}\right)^{2}-\left(\frac{\bar{y}}{q \sqrt{1-\left(n_{1} / n_{2}\right)^{2}}}\right)^{2}=1 .
$$

The zero-distance phase front of a plane refracting surface is a conic section centered at the origin 0 . In polar coordinates, (17) takes the form

$$
r=\frac{l}{\varepsilon \cdot \cos \theta-1}=\frac{q\left(n_{2} / n_{1}-1\right)\left(1-n_{1} / n_{2}\right)}{\left(n_{2} / n_{1}\right) \cdot \cos \theta-1},
$$

where $\varepsilon \equiv \sqrt{1+b^{2} / a^{2}}=\sqrt{1+\left(1-\left(n_{1} / n_{2}\right)^{2}\right) /\left(n_{1} / n_{2}\right)^{2}}=$ $n_{2} / n_{1}$ and $l \equiv b^{2} / a=q(1-1 / \varepsilon)(\varepsilon-1)$.

The source is located at the focus of the conic surface.

Thus, we have the following.

If $n_{2}>n_{1}$ (the refraction occurs from a less dense medium to a more dense medium), the zero-distance phase front of the plane refracting surface is a hyperbolic surface (Figure 7(a)).

If $n_{2}<n_{1}$ (the refraction occurs from a more dense medium to a less dense medium), the zero-distance phase front of the plane refracting surface is an elliptical surface (Figure 7(b)). 


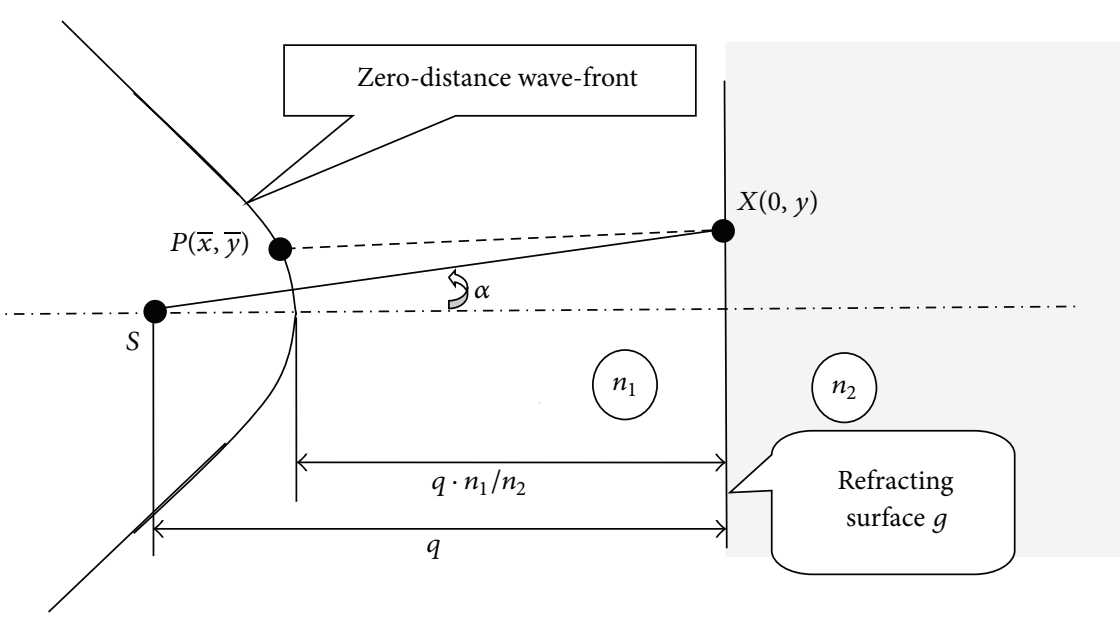

(a)

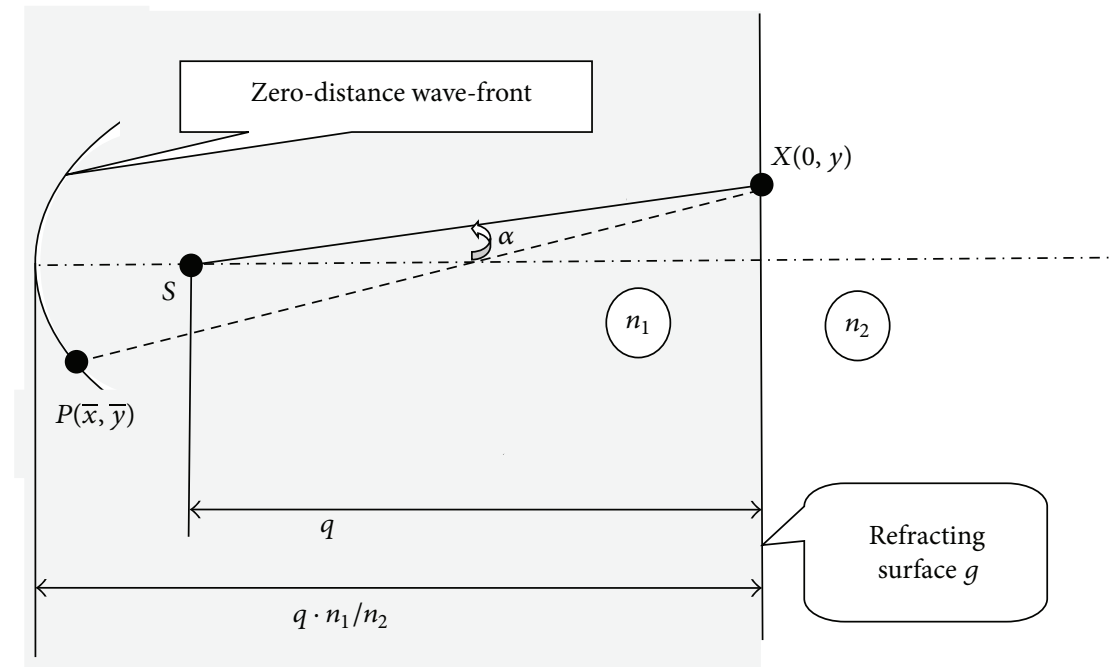

(b)

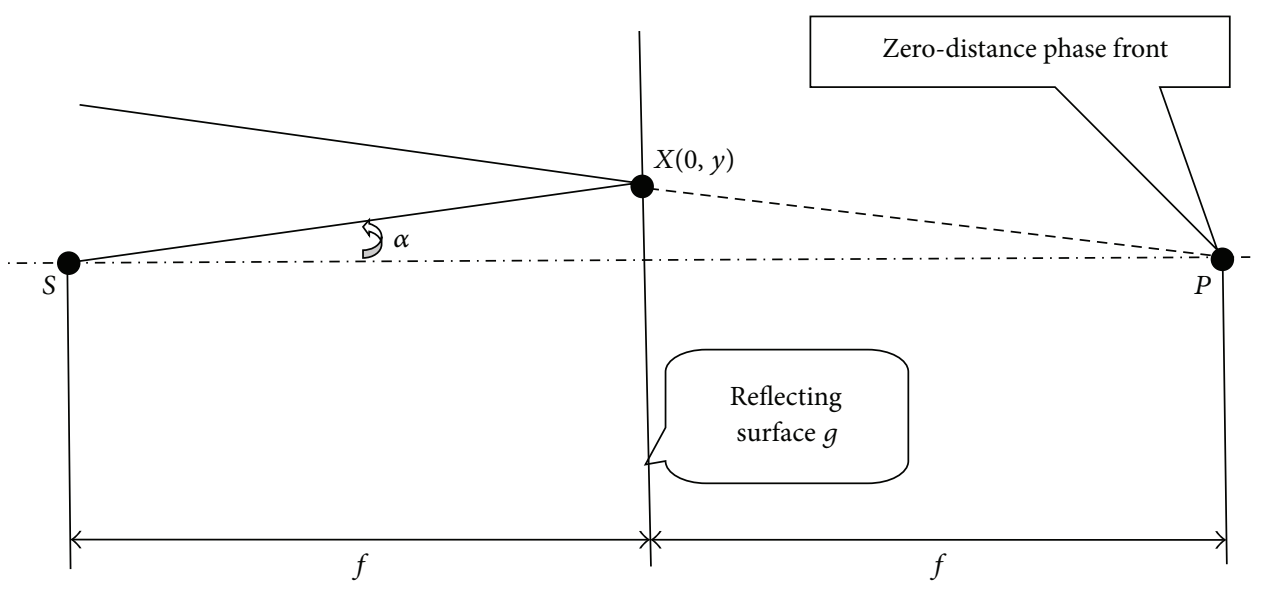

(c)

FIGURE 7: Refraction of rays from a point $S$ on a plane surface $g$ : (a) $n<n^{\prime}$; (b) $n>n^{\prime}$. (c) Reflection of rays from a point $S$ on a plane surface g. 


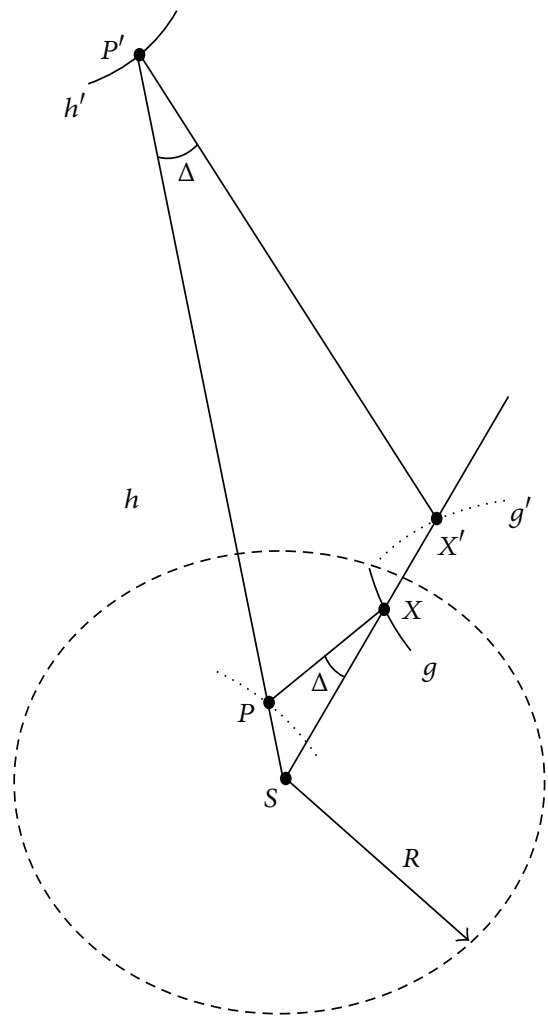

(a)

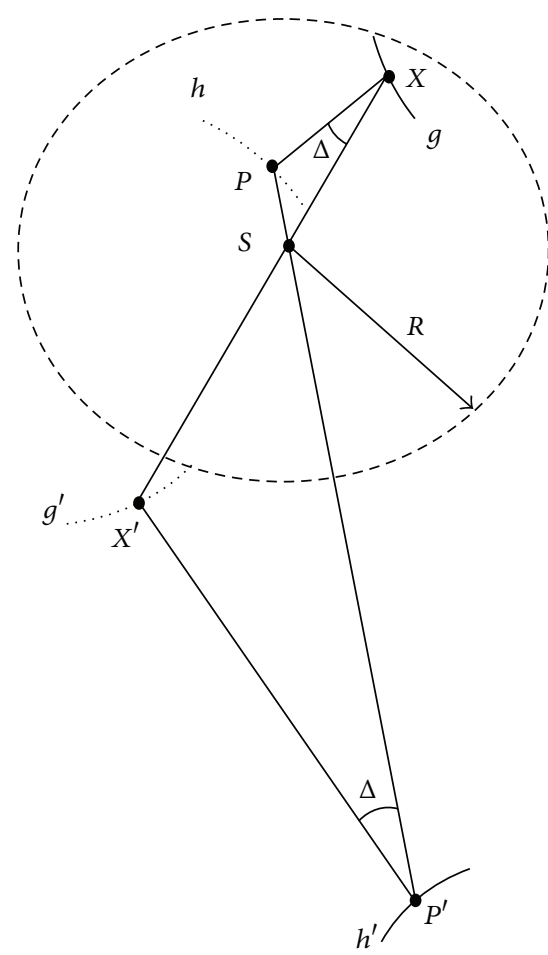

(b)

Figure 8: Illustration of Damien's inversion theorem: (a) $S X \cdot S X^{\prime}=R^{2}=S P \cdot S P^{\prime}$ (positive inverse); (b) $S X \cdot S X^{\prime}=-R^{2}=S P \cdot S P^{\prime}$ (negative inverse).

It is known that if $n_{1}=1$ and $n_{2}=-1$, the zero-distance phase front of the plane reflecting surface is a point $(f, 0)$.

\section{Damien's Inversion Theorem}

Recollect the definition of inversion $[4,6-9,13]$ : the inverse of a point $X$ with respect to a fixed circle with the center $S$ and the radius $R$ is the point $X^{\prime}$ which lies on the ray $S X$ and $S X \cdot S X^{\prime}=R^{2}$ (positive inverse) or $S X \cdot S X^{\prime}=-R^{2}$ (negative inverse). Thus, in polar coordinates, the inverse of the point $(r, \theta)$ is $(\rho, \theta)[13]$, where $\rho=R^{2} / r$ (positive inverse) or $\rho=$ $-R^{2} / r$ (negative inverse).

Damien's theorem [4, 6-10] states the following: if a surface $h$ is a zero-distance phase front of the refracting surface $g$ with respect to a point source $S$, then the inversion in a circle centered on $S$ leads to the transformation of $g$ into a surface $g^{\prime}$ and $h$ into a surface $h^{\prime}$, so that $g^{\prime}$ is the zerodistance phase front for the refracting surface $h^{\prime}$ with respect to the same point source $S$ and for the same refraction indexes (Figure 8). A refracting surface inverts into a zero-distance phase front and vice versa.

Let us prove this theorem. The inversion in the circle centered at $S$ leads to the transformation of $g$ into $g^{\prime}$ and $h$ into $h^{\prime}$. A straight line passing through points $S$ and $X$ intersects the surface $g^{\prime}$ at the point $X^{\prime}$. A straight line passing through points $S$ and $P$ intersects the surface $h^{\prime}$ at the point $P^{\prime}$. As points $X$ and $X^{\prime}$ and points $P$ and $P^{\prime}$ are inverse points with respect to the same inversion circle, they are connected by the following relation:

$$
\begin{aligned}
& S X \cdot S X^{\prime}=R^{2}=S P \cdot S P^{\prime}, \quad(\text { Figure } 8(\mathrm{a})) \\
& S X \cdot S X^{\prime}=-R^{2}=S P \cdot S P^{\prime}, \quad(\text { Figure } 8(\mathrm{~b})),
\end{aligned}
$$

where $R$ is the radius of the inversion circle.

Let us suppose that the surfaces $g$ and $h^{\prime}$ are refracting surfaces separating optically homogeneous media with refraction indexes $n_{1}$ and $n_{2}$ (the point source $S$ within the first medium). Let us consider triangles $\triangle X S P$ and $\triangle X^{\prime} S P^{\prime}$, where $\angle X S P=\angle X^{\prime} S P^{\prime}$. According to (19a) and (19b), the lengths of the sides of the triangle $\triangle X S P$ are proportional to the lengths of the sides of the triangle $\triangle X S P$. Thus, the triangles $\triangle X S P$ and $\triangle X^{\prime} S P^{\prime}$ are similar and $\angle S X P=$ $\angle S P^{\prime} X^{\prime}=\Delta$; that is, $\beta-\alpha=\beta^{\prime}-\alpha^{\prime}$. By lemma for Snell's law, the angles of incidence at the refracting surfaces $g$ and $h^{\prime}$ are the same: $\alpha=\alpha^{\prime}$. From (4) follows the proportionality $|S X|$ : $|X P|=\left|S P^{\prime}\right|:\left|P^{\prime} X^{\prime}\right|=-\left(n_{2}: n_{1}\right)$, so, taking into account definition (2), the curves $h$ and $g^{\prime}$ are the zero-distance phase fronts for refracting surfaces $g$ and $h^{\prime}$, respectively.

Note that if the circle of inversion has the radius $R=$ $q \sqrt{1-n_{1} / n_{2}}$ and the center at the point source $S$, the 


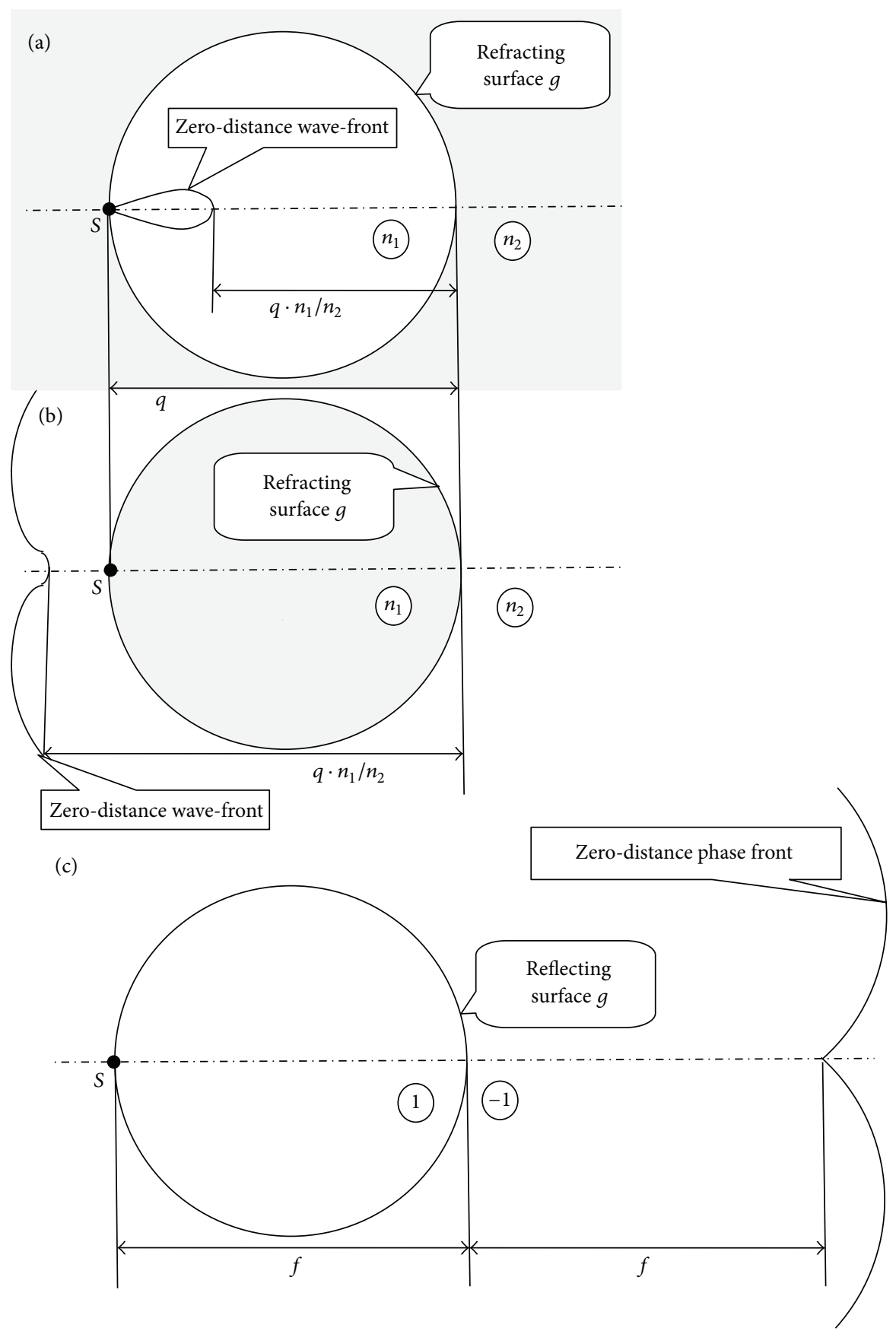

FIGURE 9: "Designing" the zero-distance phase front for spherical optical surfaces passing through the source $S$ : (a) refracting surface $\left(n_{1}<n_{2}\right)$ $\Rightarrow$ the limacon of Pascal with $\varepsilon>0$; (b) refracting surface $\left(n_{1}>n_{2}\right) \Rightarrow$ the limacon of Pascal with $\varepsilon<0$; (c) reflecting surface $\left(n_{1}=1, n_{2}=-1\right)$ $\Rightarrow$ the cardioids.

refracting surface and the zero-distance phase front will be swapped. Similarly, if the circle of inversion has the radius $R=q \sqrt{2}$ and the center at the point source $S$, the reflecting surface and the zero-distance phase front will be swapped too.

In Figure 9 the inversion of the plane zero-distance phase front $r=q\left(1-n_{1} / n_{2}\right) / \cos \theta$ with respect to a point source $S$ and the radius $R=q \sqrt{1-n_{1} / n_{2}}$ is a circle $\rho=q(1-$ $\left.n_{1} / n_{2}\right) \cos \theta$ passing through the source $S$, and the inversion of the conic refracting surface, (6), is the limacon of Pascal $(\rho, \theta)[4,6-9]$, where

$$
\begin{aligned}
\rho & =\frac{q^{2}\left(1-n_{1} / n_{2}\right)}{q\left(1-n_{2} / n_{1}\right)}\left(1-\frac{n_{2}}{n_{1}} \cdot \cos \theta\right) \\
& =q \frac{\left(1-n_{1} / n_{2}\right)}{\left(1-n_{2} / n_{1}\right)}\left(1-\frac{n_{2}}{n_{1}} \cdot \cos \theta\right) .
\end{aligned}
$$




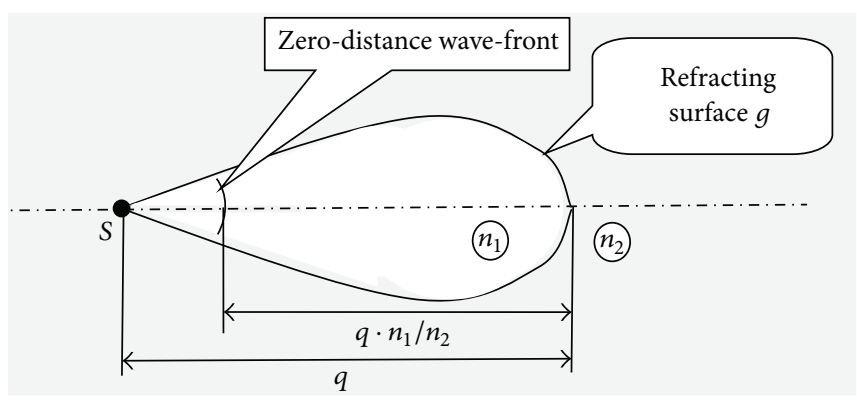

(a)

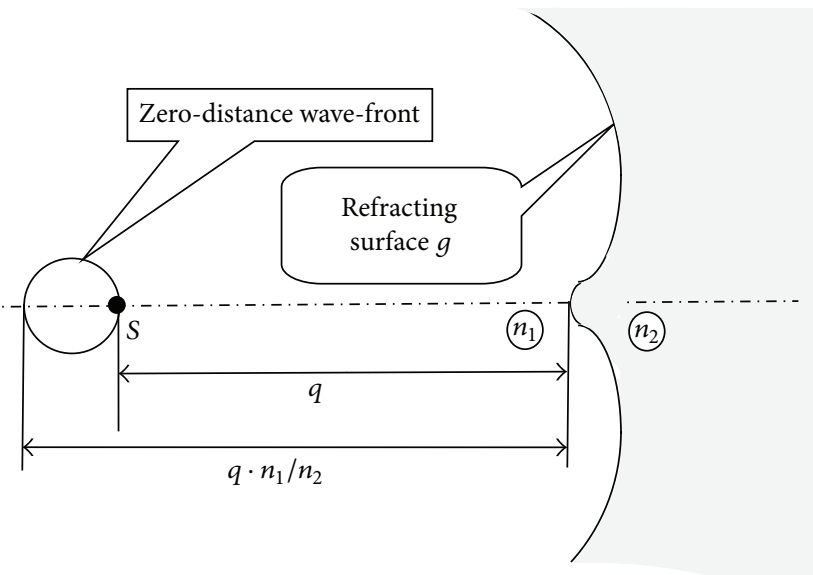

(b)

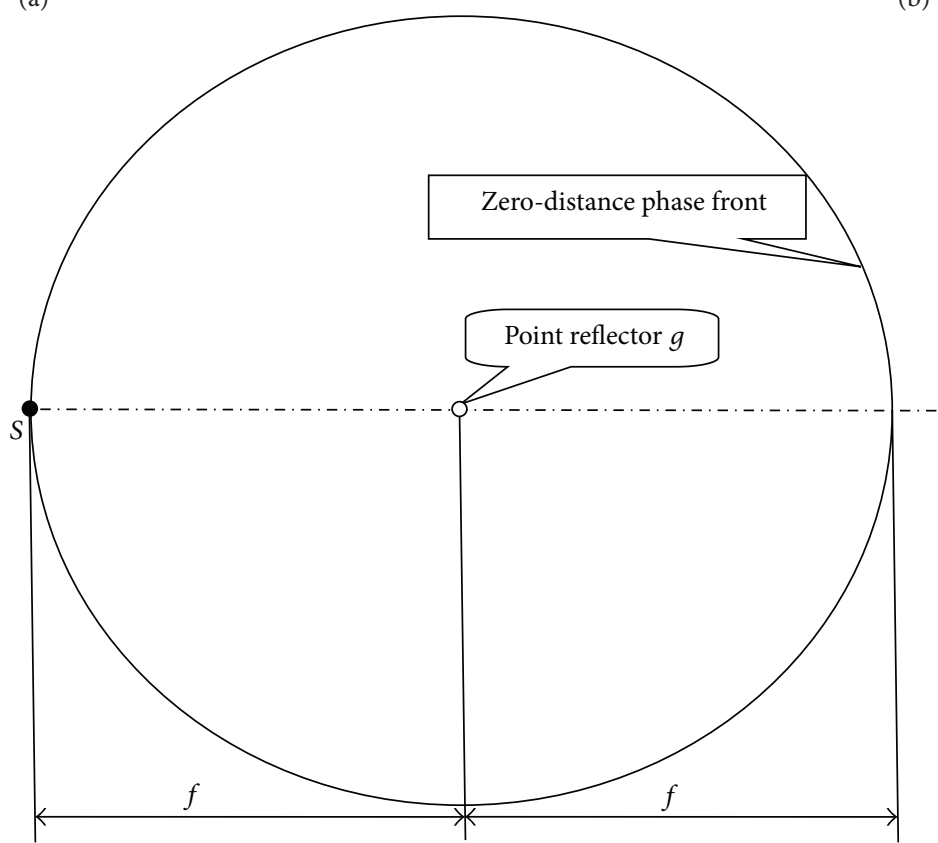

(c)

FIGURE 10: "Designing" the optical surfaces for the spherical zero-distance phase fronts passing through the source $S$ : (a) the refracting surface $n_{1}<n_{2} \Rightarrow$ the limacon of Pascal with $\varepsilon>0$; (b) the refracting surface $n_{1}>n_{2} \Rightarrow$ the limacon of Pascal with $\varepsilon<0$; (c) the reflecting surface $\Rightarrow$ the point reflector (small mirror sphere).

Thus, according to Damien's inversion theorem, the pair "the conic refracting surface-the plane zero-distance phase front" (Figures 5(a) and 5(b)) corresponds to the dual pair "the spherical refracting surface-the zero-distance phase front in the form of the limacon of Pascal" (Figures 9(a) and 9(b)).

The inversion of the plane zero-distance phase front $r=$ $2 f / \cos \theta$ is a circle $\rho=f \cdot \cos \theta$ passing through the source $S$. The inversion of the parabolic reflecting surface, (11), with respect to a point source $S$ and the radius $R=f \sqrt{2}$, is the cardioid $(\rho, \theta)[4,6-9]$, where

$$
\rho=\frac{2 f^{2}}{2 f}(1+\cos \theta)=f(1+\cos \theta) .
$$

Thus, according to Damien's inversion theorem, the pair "the parabolic reflecting surface-the plane zero-distance phase front" (Figure 5(c)) corresponds to the dual pair "the spherical reflecting surface-the zero-distance phase front in the form of the cardioid" (Figure 9(c)).

In Figure 10 the inversion of the plane refracting surface $r=d / \cos \theta$ with respect to a point source $S$ and the radius $R=d \sqrt{1-n_{1} / n_{2}}$ is a circle $\rho=d \sqrt{1-n_{1} / n_{2}} \cos \theta$ passing through the source $S$, and the inversion of the conic refracting surface (18) is the limacon of Pascal $(\rho, \theta)[4,6-9]$, where

$$
\begin{aligned}
\rho & =\frac{d^{2}\left(1-n_{1} / n_{2}\right)}{d\left(1-n_{2} / n_{1}\right)\left(1-n_{1} / n_{2}\right)}\left(1-\frac{n_{2}}{n_{1}} \cdot \cos \theta\right) \\
& =\frac{d}{\left(1-n_{2} / n_{1}\right)}\left(1-\frac{n_{2}}{n_{1}} \cdot \cos \theta\right) .
\end{aligned}
$$


Thus, according to Damien's inversion theorem, the pair "the plane refracting surface-the conic zero-distance phase front" (Figures 7(a) and 7(b)) corresponds to the dual pair "the refracting surface in the form of the limacon of Pascal-the spherical zero-distance phase front" (Figures 10(a) and 10(b)).

According to Damien's inversion theorem, the pair "the plane reflecting surface-the point zero-distance phase front" (Figure $7(\mathrm{c})$ ) corresponds to the dual pair "the point reflecting surface-the spherical zero-distance phase front" (Figure 10(c)).

\section{Conclusion}

(1) A simple proof of Damien's theorem has been proposed.

(2) A systematic study of the dual pairs "the optical surface-the zero-distance phase front" has been performed for both refractive and reflective optical surfaces.

\section{Conflict of Interests}

The author declares that there is no conflict of interests regarding the publication of this paper.

\section{References}

[1] Projective geometry, Wikipedia, the free encyclopedia, http://en .wikipedia.org/wiki/Projective_geometry.

[2] A. V. Gitin, Duality principle in optics, radiometry and illumination engineering [Doctoral dissertation], St. Petersburg, S.I. Vavilov State Optical Institute, 1998, (Russian), http://www .dissercat.com/content/printsip-dualnosti-v-optike-radiometrii-i-svetotekhnike.

[3] A. V. Gitin, "Iconics-a comprehensive approach to optics," Journal of Optical Technology, vol. 65, no. 2, pp. 141-142, 1998.

[4] S. Cornbleet, "Geometrical optics reviewed: a new light on an old subject," Proceedings of the IEEE, vol. 71, no. 4, pp. 471-502, 1983.

[5] J. E. Eaton, "The zero phase-front in microwave optics," Transactions of the IRE Professional Group on Antennas and Propagation, vol. 1, no. 1, pp. 38-41, 1952.

[6] S. Cornbleet, Microwave Optics: The Optics of Microwave Antenna Design, Academic Press, London, UK, 1976.

[7] S. Cornbleet, Microwave and Optical Ray Geometry, John Wiley \& Sons, Chichester, UK, 1984.

[8] S. Cornbleet, Microwave and Geometrical Optics, Academic Press, San Diego, Calif, USA, 1994.

[9] S. Cornbleet, "New geometrical method for the design of optical systems," IEE Journal on Microwaves, Optics and Acoustics, vol. 3, no. 2, pp. 78-84, 1979.

[10] R. Damien, Theoreme sur les surfaces d'onde en optique geometrique, Gauthier-Villars, Paris, France, 1955.

[11] O. N. Stavroudis, "Refraction of wavefronts: a special case," Journal of the Optical Society of America, vol. 59, no. 1, pp. 114$115,1969$.

[12] M. Born and E. Wolf, Principles of Optics, Cambridge University Press, Cambridge, UK, 1999.
[13] Wikipedia, the free encyclopedia, Inverse curve, http://en .wikipedia.org/wiki/Inverse_curve. 

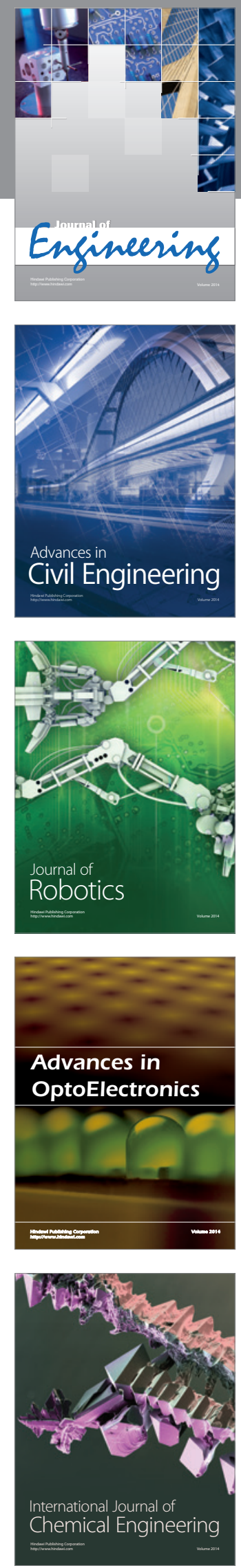

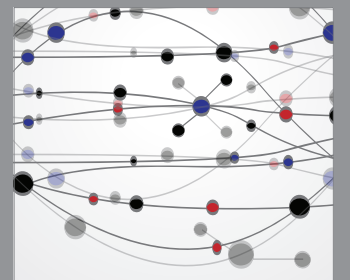

The Scientific World Journal
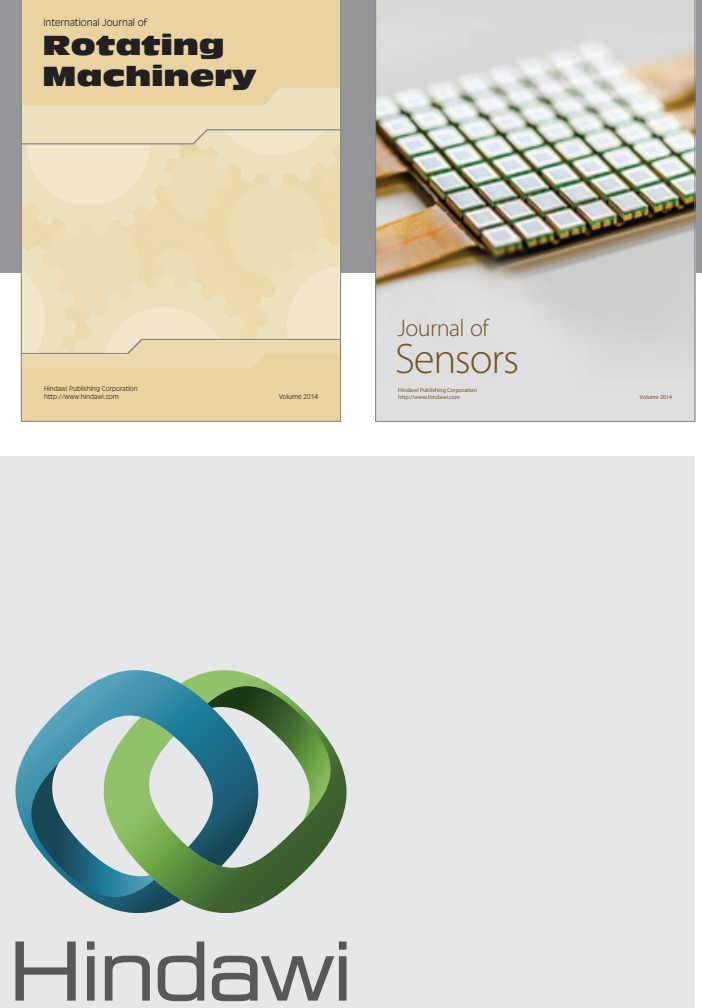

Submit your manuscripts at http://www.hindawi.com
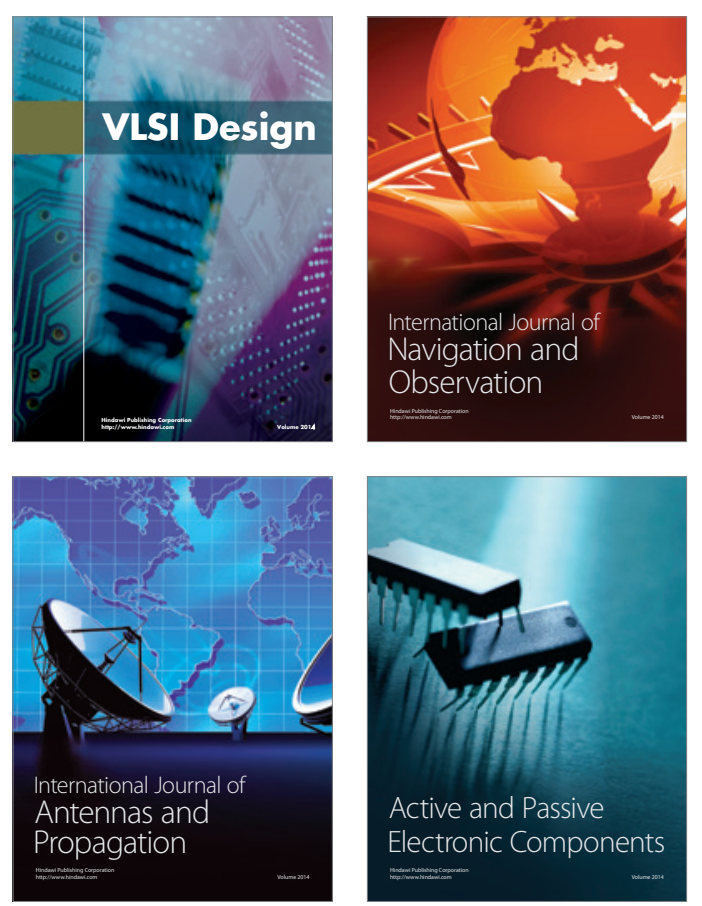
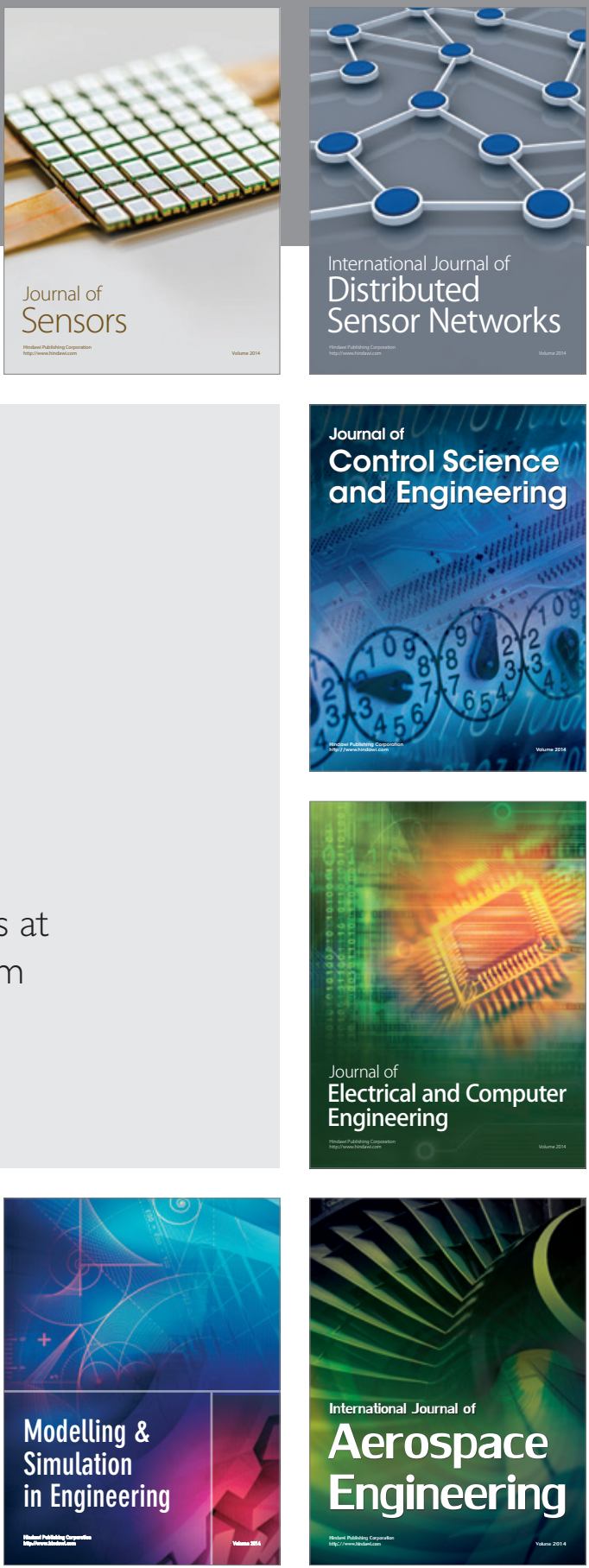

Journal of

Control Science

and Engineering
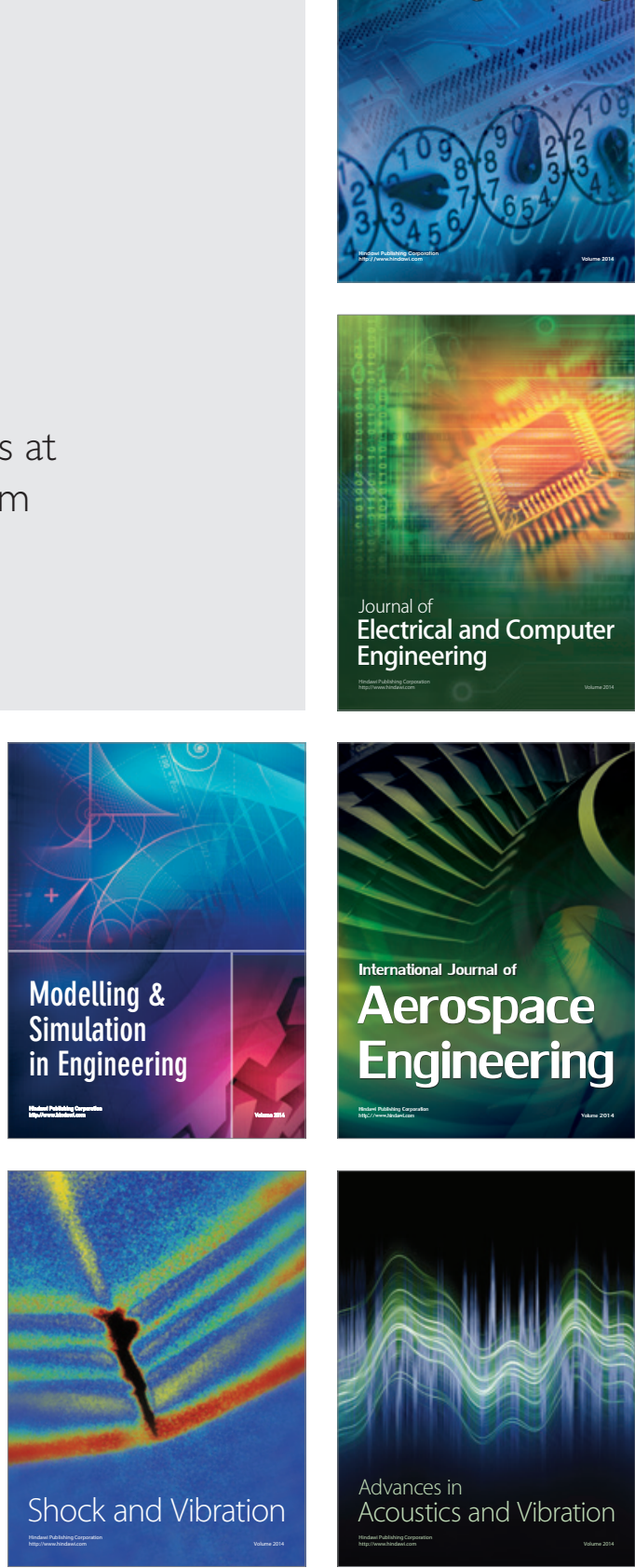